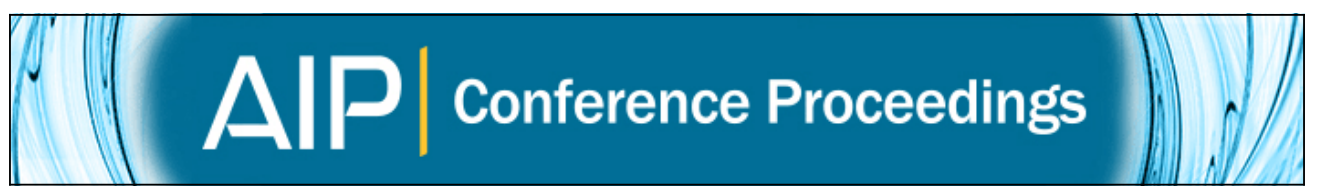

\title{
Uniqueness of the Maximal Helicoid
}

I. Fernández and Francisco J. López

Citation: AIP Conference Proceedings 1023, 153 (2008); doi: 10.1063/1.2958167

View online: http://dx.doi.org/10.1063/1.2958167

View Table of Contents:

http://scitation.aip.org/content/aip/proceeding/aipcp/1023?ver=pdfcov

Published by the AIP Publishing

\section{Articles you may be interested in}

Exploring the transition from special to general relativity

Am. J. Phys. 77, 434 (2009); 10.1119/1.3088883

MicroReviews by the Book Review Editor: Relativity: An Introduction to Special and General Relativity (3rd ed.): Hans Stephani

Phys. Teach. 43, 624 (2005); 10.1119/1.2136478

General Relativity: A Geometric Approach

Am. J. Phys. 68, 583 (2000); 10.1119/1.19490

Flat wormholes from cosmic strings

J. Math. Phys. 38, 5807 (1997); 10.1063/1.532167

Gravitational energy of conical defects

J. Math. Phys. 38, 458 (1997); 10.1063/1.531827 


\title{
Uniqueness of the Maximal Helicoid
}

\author{
I. Fernández* and Francisco J. López ${ }^{\dagger}$ \\ *Departamento de Matemática Aplicada I, E.T.S. de Ingeniería Informática, Universidad de \\ Sevilla, Avenida Reina Mercedes s/n, 41012 - Sevilla, SPAIN \\ ${ }^{\dagger}$ Departamento de Geometría y Topología, Facultad de Ciencias, Universidad de Granada, \\ 18071-Granada, SPAIN
}

\begin{abstract}
The purpose of this paper is to briefly outline the uniqueness of the helicoid and Enneper's surface among maximal surfaces in the Lorentz-Minkowski space $\mathbf{R}_{\mathbf{1}}^{\mathbf{3}}$.
\end{abstract}

Keywords: Spacelike, Maximal Immersion, Properly Embedded

PACS: $02.40 .-\mathrm{k}, 02.40 . \mathrm{Vh}, 02.30 . \mathrm{Xx}, 04.20 . \mathrm{q}$

\section{INTRODUCTION}

The Euclidean helicoid $\mathscr{H}_{0}$ is the third minimal surface in $\mathbf{R}^{\mathbf{3}}$ to be known, after the plane and the catenoid (J. B. Meusnier, 1776). It is also the only minimal surface foliated by straight lines, besides the plane (Catalan, 1842), and its name derives from its similarity to the helix (see Figure 1, $(a)$ ). A surface in $\mathbf{R}^{\mathbf{3}}$ is said to be minimal if it represents a local minima for the area functional, or equivalently, if it has zero mean curvature. Topologically, $\mathscr{H}_{0}$ is a properly embedded surface in $\mathbf{R}^{\mathbf{3}}$ homeomorphic to the plane, and can be described by the following parametric equations:

$$
x=\rho \cos (\theta), \quad y=\rho \sin (\theta), \quad z=\theta, \quad \text { where }(\rho, \theta) \in \mathbf{R}^{2} .
$$

It appears in nature in both macroscopic and microscopic scale (e.g., spiral galaxies, DNA structure), and has inspired architecture and art across the world (e.g., the helicoid of Caracas (Caracas, Venezuela) and the Sagrada Familia (Barcelona, Spain)).

The geometry of $\mathscr{H}_{0}$ is concentrated in the axis, which is the vertical line of points with horizontal normal vector and maximal Gaussian curvature. Figure 1, $(b)$ corresponds to a half of the helicoid bounded by the vertical axis.

By definition, the Lorentzian helicoid $\mathscr{H}$ is the spacelike part of the Euclidean one when viewed in the Lorentz-Minkowski space $\mathbf{R}_{\mathbf{1}}^{\mathbf{3}}$. Recall that, by definition, $\mathbf{R}_{\mathbf{1}}^{\mathbf{3}}$ is the space $\mathbf{R}^{3}$ endowed with the indefinite metric of signature $(1,1,-1)$ given by $\langle x, y\rangle=$ $x_{1} y_{1}+x_{2} y_{2}-x_{3} y_{3}$. To be more precise, $\mathscr{H}$ coincides with the relative complement of the rigid circular cylinder $\left\{(x, y, z): x^{2}+y^{2}+z^{2} \leq 1\right\}$ in half of the Euclidean helicoid, as suggested in Figure 2. Amazingly, $\mathscr{H}$ also represents a critical point for the area functional associated to variations by spacelike surfaces in $\mathbf{R}_{\mathbf{1}}^{\mathbf{3}}$, but in this case it is a local maximum. This kind of surfaces are said to be maximal, and like in the Euclidean case, they are characterized by the property of having zero mean curvature. As a matter of fact, O. Kobayashi [3] proved that spacelike planes and Lorentzian helicoids are the only maximal surfaces which are also minimal surfaces with respect to the Euclidean metric on the ambient 3-space. 


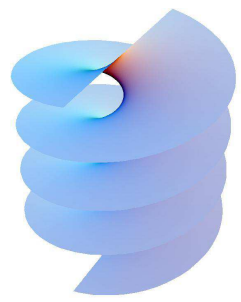

(a)

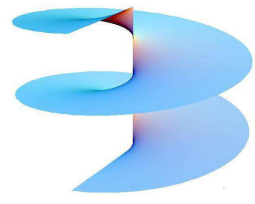

(b)

FIGURE 1. (a) The Euclidean Helicoid; (b) Half of the Euclidean Helicoid.
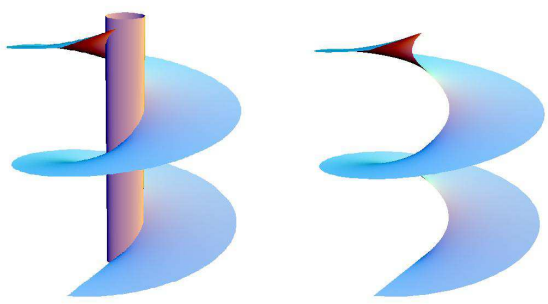

FIGURE 2. The Lorentzian Helicoid.

The boundary behavior of $\mathscr{H}$ focuses on relevant geometrical information of the surface. Indeed, its boundary consists of a lightlike helix with infinite rotation number which projects onto the unit circle in $\{z=0\}$. By the rotation number we mean the change of the tangent angle it its orthogonal projection on the plane $\{z=0\}$.

Furthermore, and roughly speaking, the boundary curve $\partial(\mathscr{H})$ is an integral curve of the intrinsic gradient of the third coordinate function. This condition is equivalent to the property that any conformal embedding $X: M \equiv\{\operatorname{Im}(\mathrm{z}) \geq 0\} \rightarrow \mathbf{R}_{\mathbf{1}}^{\mathbf{3}}$ of the Lorentzian helicoid folds back at $\partial(M)$, that is to say, $X$ extends harmonically to the double of $M$ being invariant under the mirror involution. Maximal surfaces with regular lightlike boundary and satisfying this symmetry property are said to have lightlike boundary of mirror symmetry, or simply * maximal surfaces.

Another interesting example of properly embedded *maximal surface in $\mathbf{R}_{\mathbf{1}}^{\mathbf{3}}$ is the so called Lorentzian Enneper surface $\mathscr{E}=\left\{(x, y, t): 32(y-t)^{3}-3(y+t)+24(y-t) x=0\right\}$ described by Kobayashi in [3]. Unlike the Lorentzian helicoid, the Lorentzian Enneper surface has well defined lightlike tangent plane at infinity and its boundary does not spiral indefinitely (that is to say, it has finite rotation number).

\section{MAXIMAL MULTIGRAPHS}

Properly embedded *maximal surfaces with connected boundary are multigraphs in a generalized sense. Indeed, if $M$ is a such surface then its boundary $\partial(M)$ is a lightlike regular curve satisfying the cone property (i.e., for any $p \in \partial(M), \partial(M)-\{p\}$ lies in 


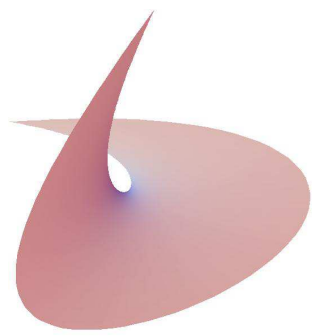

FIGURE 3. The Lorentzian Enneper surface.

the interior of the lightcone with vertex $p), \partial(M)$ projects onto a locally convex planar curve $\gamma$ in $\{z=0\}$ and the orthogonal projection $\pi: M \rightarrow\{z=0\}$ is locally inyective. In particular, $M$ is simply connected and can be imagined as a series of pieces (or leaves) matching like the steps of a stair. One can define the rotation number $\theta(M)$ of $M$ as the change of the tangent angle of $\gamma$.

On the other hand, a proper curve $\alpha \cong[0,1[$ is said to be affinely spacelike if it lies in a spacelike wedge of $\mathbf{R}_{1}^{\mathbf{3}}$ (i.e., a closed wedge foliated by spacelike half planes with the same edge), and surfaces that are disjoint from an affinely spacelike curve are said to be $\omega$-surfaces $\left(\omega^{*}\right.$-surfaces if in addition they are *maximal).

Viewing the Lorentzian helicoid as maximal multigraph, we can observe that the curvature of any of its leaves decays to zero at infinity. As a consequence:

(I) Every leaf $\mathscr{L}$ of the Lorentzian helicoid admits, when viewed as a maximal graph $u(x, y)$, the gradient estimate $\|\nabla u\|<1-\varepsilon$ on $\mathscr{L}-V$, where $\varepsilon>0$ and $V$ is a neighborhood of $\partial(\mathscr{L}) \cap \partial(\mathscr{H})$.

(II) The intrinsic metric in $\mathscr{L}-V$ is equivalent to the pull-back of the Euclidean one in $\{z=0\}$ via the orthogonal projection.

(III) The Lorentzian helicoid is $\omega^{*}$-maximal.

Returning to the general context of properly embedded *maximal surfaces with connected boundary, it is not hard to check that (I) $\Rightarrow$ (III) and (II) $\Rightarrow$ (III). Moreover, the notions of $\omega^{*}$-maximal and ${ }^{*}$ maximal are equivalent when the rotation number of the multigraph is finite (although it is expected that this occurs in the general case).

Our main result (see [2]) asserts:

Main Theorem. The Lorentzian helicoid and the Lorentzian Enneper surface are the only properly embedded $w^{*}$-maximal surfaces with connected boundary.

The family of $\omega^{*}$-maximal surfaces with non connected boundary is very vast. Therefore, the connectedness of the boundary is a crucial hypothesis: Figure 4 shows a Riemann type properly embedded maximal surface bounded by two lightlike arcs of mirror symmetry (see [5]). The authors conjecture that the theorem remains valid if we replace the hypothesis $w^{*}$-maximal for the more general one of *maximal.

The above theorem is a sort of Lorentzian compilation in the context of $\omega^{*}$-maximal surfaces of two recent Riemannian theorems. Indeed, W.H. Meeks and H. Rosenberg 


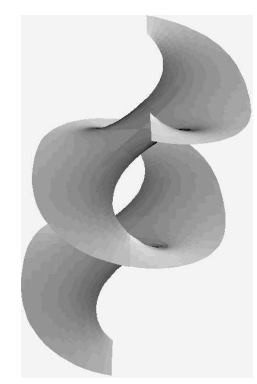

FIGURE 4. A Riemann type maximal surface.

[7] have characterized $\mathscr{H}_{0}$ as the unique non flat properly embedded simply connected minimal surface in $\mathbf{R}^{\mathbf{3}}$, and likewise, J. Pérez [6] has proved that half of the Enneper minimal surface is the only properly embedded non flat oriented stable minimal surface bounded by a straight line and having quadratic area growth.

\section{SKETCH OF THE PROOF}

The proof strategy of the theorem has essentially three ingredients: Colding-Minicozzi theory for properly embedded maximal surfaces, Meeks-Rosenberg theory for properly embedded maximal surfaces and a finiteness theorem for disjointly supported maximal graphs. For more details we refer to [2].

The original Colding-Minicozzi theory is devoted to the study of the asymptotic behavior of properly embedded minimal surfaces at infinity. In other words, to understand the geometry of any homothetical blow down of a such surface [1].

The homothetical blow downs of $\mathscr{H}$ are degenerated planar multigraphs with a singular point, that is to say, the leaves of $\mathscr{H}$ accumulate at the plane $\{z=0\}$ and the entire helicoid is sucked into the origin. A similar analysis can be done with the Lorentzian Enneper surface, but in this case the blow down limit is a lightlike plane. The deepest part of our Main Theorem consists of proving the following theorem:

Theorem 1. If $M$ is a $w^{*}$-maximal surface with connected boundary, then any homothetical blow down of $M$ converges to a plane $\Sigma_{\infty}$ as degenerated multigraph with a singular point. Furthermore:

- The blow down plane $\Sigma_{\infty}$ is spacelike if and only if $\partial(M)$ spirals indefinitely to the top and to the bottom. In particular, $\theta(M)=+\infty$.

- $\Sigma_{\infty}$ is lightlike if and only if $\theta(M)<+\infty$.

This result is quite technical and requires some regularity theorems for solutions of the maximal surface equation and parabolicity criteria for maximal surfaces.

Meeks-Rosenberg theory [7] consists of establishing under what geometrical and topological conditions the Gauss map of the surface omits a given direction in the sphere. In this setting the following theorem plays a fundamental role: 
Theorem 2. Let $M$ be a properly embedded $\omega^{*}$ maximal surface with connected boundary. Then, there exists an exhaustion of $\mathbf{R}_{1}^{\mathbf{3}}$ by closed non compact regions $\left\{C_{n}\right\}_{n \in \mathbf{N}}$ (not necessarily cylinders), and a sequence of foliations $\left\{\mathscr{F}_{n}\right\}_{n \in \mathbf{N}}$ such that:

- $\mathscr{F}_{n}$ is a foliation of $C_{n}$ by maximal discs with boundary lying in $\partial\left(C_{n}\right)$.

- Any disc in $\mathscr{F}_{n}$ and $M$ meet transversally into a Jordan arc.

- $\left\{\mathscr{F}_{n}\right\}_{n \in \mathbf{N}}$ converges to the foliation of $\mathbf{R}_{\mathbf{1}}^{\mathbf{3}}$ by planes parallel to $\Sigma_{\infty}$.

By a Morse theory argument we can prove the following corollary:

Corollary 1. $M$ omits the normal direction to $\Sigma_{\infty}$.

Finally, the finiteness theorem deals with the question of how many disjointly supported maximal graphs over the plane $\{z=0\}$ can exist. Inspired by the work [4] by $\mathrm{Li}$ and Wang for minimal graphs, we have obtained the following result:

Theorem 3. Let $\left\{G_{i}\right\}_{i=1}^{k}$ be a set of $k$ maximal simply connected graphs in $\mathbf{R}_{\mathbf{1}}^{\mathbf{3}}$ defined by the functions $\left\{u_{i}\right\}_{i=1}^{k}$ with disjoint supports in $\{z=0\}$. Let us assume that $\left\|\nabla u_{i}\right\|_{0} \leq 1-\varepsilon$, for any $i=1, \ldots, k$, where $\varepsilon>0$. Then $k \leq \frac{8}{\varepsilon(2-\varepsilon)}$.

We use this theorem and the previous ingredients to infer that $M \cap \Sigma$ consits of finitely many proper arcs, and with a little more effort, of a unique proper arc, for any plane $\Sigma$ parallel to the blow down plane $\Sigma_{\infty}$.

The above results let us control the conformal structure and Weierstrass data of any $\omega^{*}$-maximal surface with connected boundary, leading to our uniqueness result.

\section{ACKNOWLEDGMENTS}

We are indebted to Prof. R. Souam for many useful conversations about this subject.

\section{REFERENCES}

1. T. H. Colding and W. P. Minicozzi: The space of embedded minimal surfaces of fixed genus in a 3-manifold IV; Locally simply connected. Ann. of Math., 160 (2004), 573-615.

2. I. Fernández, F. J. López: On the uniqueness of the helicoid and Enneper's surface in the LorentzMinkowski space $\mathbf{R}_{\mathbf{1}}^{\mathbf{3}}$. Preprint.

3. O. Kobayashi: Maximal surfaces in the 3-dimensional Minkowski space $\mathbf{R}_{\mathbf{1}}^{\mathbf{3}}$. Tokyo J. Math., 6 (1983), no. 2, p.p. 297-309.

4. P. Li and J. Wang: Finiteness of disjoint minimal graphs. Math. R. Letters, 6 (2002), no. 5-6, 771-778.

5. F. J. López, R. López and R. Souam: Maximal surfaces of Riemann type in Lorentz-Minkowski space L $^{3}$. Michigan J. of Math., 47 (2000), 469-497.

6. J. Pérez: Stable embedded minimal surfaces bounded by a straight line. Calculus of Variations and Partial Differential Equations, 29, Number 2, June 2007, 267-279(13).

7. W. H. Meeks III and H. Rosenberg: The uniqueness of the helicoid. Ann. Math., 161 (2005), 727-758. 